\title{
Exercise effects on sleep physiology
}

\section{Sunao Uchida*, Kohei Shioda, Yuko Morita, Chie Kubota, Masashi Ganeko and Noriko Takeda}

Faculty of Sport Sciences, Waseda University Graduate School, Japan Society for the Promotion of Science, Waseda University, Tokorozawa, Japan

\section{Edited by:}

V. Mohan Kumar, Sree Chitra Tirunal Institute for Medical Sciences and

Technology, India

\section{Reviewed by:}

Bindu M. Kutty, National Institute of Mental Health and Neurosciences, India

V. Mohan Kumar, Sree Chitra Tirunal Institute for Medical Sciences and

Technology, India

${ }^{*}$ Correspondence:

Sunao Uchida, Faculty of Sport Sciences, Waseda University,

2-579-15 Mikajima, Tokorozawa 359-1192, Japan.

e-mail: sunao@waseda.jp
This mini-review focuses on the effects of exercise on sleep. In its early days, sleep research largely focused on central nervous system (CNS) physiology using standardized tabulations of several sleep-specific landmark electroencephalogram (EEG) waveforms. Though coarse, this method has enabled the observation and inspection of numerous uninterrupted sleep phenomena. The research on the effects of exercise on sleep began, in the 1960s, with a focus primarily on sleep related EEG changes (CNS sleep). Those early studies found only small effects of exercise on sleep. However, more recent sleep research has explored not only CNS functioning, but somatic physiology as well. Sleep should be affected by daytime exercise, as physical activity alters endocrine, autonomic nervous system (ANS), and somatic functions. Since endocrinological, metabolic, and autonomic changes can be measured during sleep, it should be possible to assess exercise effects on somatic physiology in addition to CNS sleep quality, evaluated by standard polysomnographic (PSG) techniques. Additional measures of somatic physiology have provided enough evidences to conclude that the auto-regulatory, global regulation of sleep is not the exclusive domain of the CNS, but it is heavily influenced by inputs from the rest of the body.

Keywords: exercise, sleep, EEG, body temperature, GH, cortisol, BDNF, fitness

\section{SLEEP RESEARCH HAS BEEN FOCUSED ON CENTRAL NERVOUS SYSTEM SLEEP}

In its early days, sleep research largely focused on central nervous system (CNS) physiology by tabulating several sleep-specific landmark electroencephalogram (EEG) waveforms, which enabled the observation and inspection of uninterrupted sleep phenomena (Davis et al., 1937). Dramatic changes in the patterns of EEG waveform distributions across the night enchanted and drove sleep researchers to pursue meaningful physiological correlates underlying these EEG "events." The subsequent discovery of rapid eye movement (REM) sleep (Aserinsky and Kleitman, 1953) stimulated research that has led to the understanding that sleep is not simply a resting state of the brain, but an active, dynamic cycling through multiple sleep-specific states. Computer analyses of sleep EEG (Feinberg et al., 1978; Uchida et al., 1999) and modern neuroimaging techniques (Kajimura et al., 1999) have enabled increasingly detailed analyses of CNS functions during sleep.

Thus, investigations of exercise effects on sleep have also been mainly focused on CNS functions during sleep. However, exercise is a complex physical activity that broadly affects somatic physiology with some persistent effects, and others limited during sleep. Review of the literature reveals that these changes have not yet been thoroughly explored. This mini-review focuses on the effects of exercise on sleep, including the effects on somatic physiology. We attempt to frame a future perspective for research in this field as well.

\section{EXERCISE EFFECTS ON CNS SLEEP}

The first scientific paper studying the effect of exercise on subsequent night sleep was written by Baekeland and Lasky (1966). In their study, 10 physically fit college students accustomed to participating in strenuous athletics of various kinds underwent three conditions that provided afternoon exercise, evening exercise, and no-exercise. Afternoon exercise resulted in significantly more slow wave sleep (SWS) stages 3 and 4 (Rechtschaffen and Kales, 1968) during the next sleep period, as compared with sleep after the no-exercise condition. After evening exercise, however, the amount of SWS fell between the "following no-exercise" and "afternoon exercise" conditions, but with no significant differences from either. Despite several drawbacks including limited control for the type (aerobic or anaerobic) and intensity of exercise, and the limited descriptions of exercise timing, the authors highlighted three essential observations that have repeatedly been confirmed in later studies. First of all, a single bout of exercise can increase the amount of subsequent SWS. Secondly, exercise shortly before going to bed could also produce a stress effect that can reduce the amount of subsequent SWS. Thirdly, relative to other studies, the amount of SWS following the no-exercise condition still exceeded prior normal values, which might be attributable to the subjects' overall fitness and exercise habits, i.e., habitual exercise may increase SWS.

Figure 1 was compiled from meta-analytic studies by Kubitz et al. (1996) and Youngstedt et al. (1997), who examined acute and chronic exercise effects on sleep (these two papers also provide a very comprehensive bibliography). Although this figure requires careful consideration due to the different effect sizes for each item, it can give a good overview of how daytime exercise affects nightly sleep.

Overall, studies of both acute and chronic exercise have found the effects of exercise on subsequent sleep include longer periods of NREM sleep stages and shorter periods of stage REM. However, the review of chronic exercise studies reveals robust and consistent data. The meta-analyses summarizing chronic exercise 

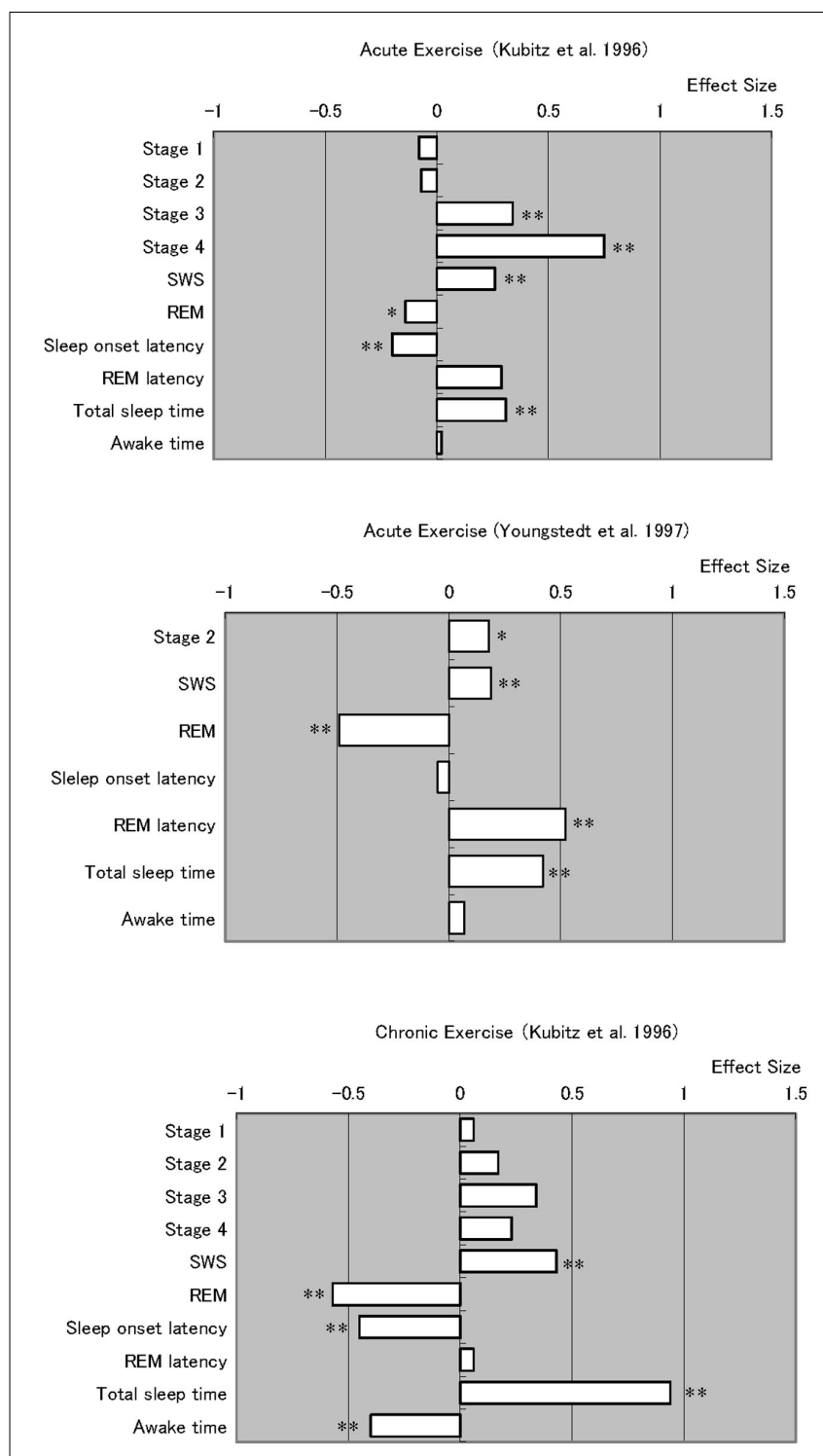

FIGURE 1 | Meta analyses of acute and chronic exercise effects on sleep parameters. Note that chronic exercise resulted larger effects on improving sleep quality, which appeared in total sleep time extension and awake time reduction. ${ }^{*} p<0.05,{ }^{*} p<0.01$.

effects reveal significantly shorter sleep onset latency, time awake after sleep onset, and significantly longer total sleep time.

As Youngstedt et al. (1997) discussed in detail, many factors contribute to the inconsistent effects of acute exercise on sleep. In their meta-analysis, they identified several moderator variables including the fitness of subjects, exercise heat load, duration and time of day, and subjects' light exposure and sleep schedule, as well as small numbers of subjects and overall study quality. Thus, studies that do not control for these factors will not give consistent results. Even so, another important difference between acute and chronic exercise is that chronic exercise substantially changes somatic functions in ways one bout of exercise does not. These somatic changes include long-term improvements in body composition, basic metabolic rate, cardiac function, glycemic

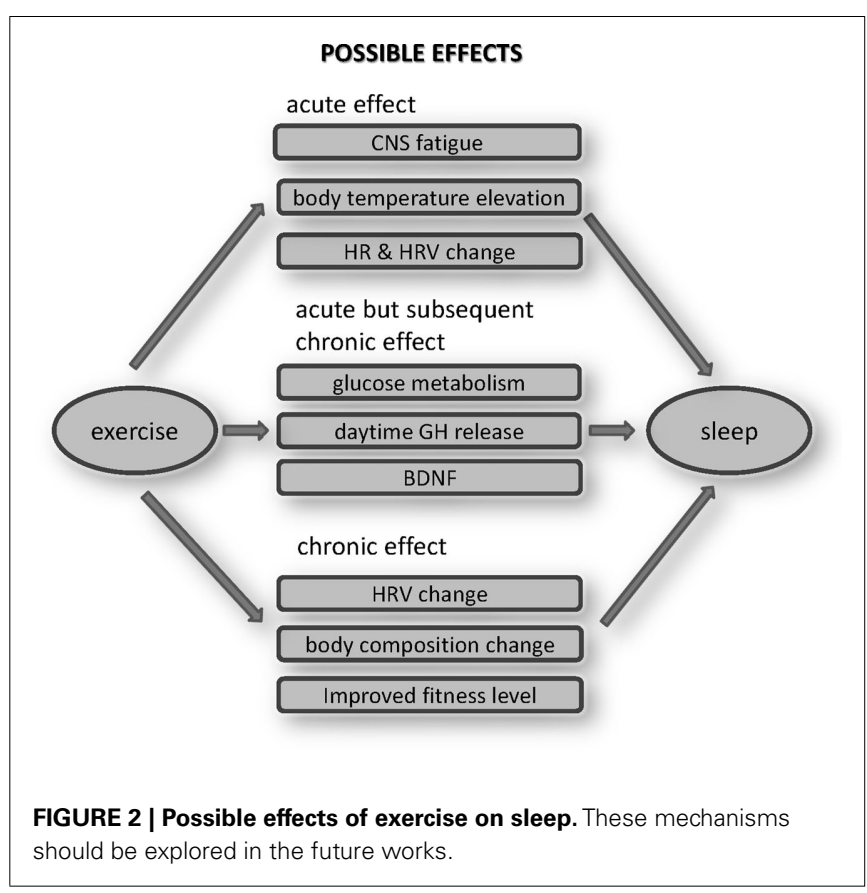

control, and immune function, as have been well studied in chronic somatic diseases (Roberts and Barnard, 2005; Pedersen and Saltin, 2006). In addition to such somatic changes, it is known that exercise also improves mood state (Blumenthal et al., 1999; Dunn et al., 2005), which can also be an additional factor in improving (or disrupting) sleep. Such long-term changes in overall health status should be considered when changes in sleep quality are evaluated in chronic exercise conditions.

\section{EXERCISE EFFECTS ON BODY TEMPERATURE DURING SLEEP}

The relationship between body temperature (BT) regulation and sleep has been well studied (Kumar, 2004). In human studies, there have been several studies which measured core BT during sleep after bouts of exercise, with a variety of inconsistent results which range from no change (Flausino et al., 2012), to BT elevation (Horne and Staff, 1983; Shioda et al., 2012).

A theoretical basis for sleep enhancement by BT elevation has been proffered by the hypothesis that SWS, which is associated with effects that reduce BT, might be promoted by higher BT before bedtime (McGinty and Szymusiak, 1990). Further research is needed to determine whether the exercise effect of enhanced SWS is due to such a mechanism.

\section{EXERCISE EFFECTS ON CARDIAC AND AUTONOMIC FUNCTION DURING SLEEP}

It was noticed in an early study that daytime exercise also increased heart rate (HR) during sleep (Walker et al., 1978), a finding that has since been reported in several studies (Roussel and Buguet, 1982; Bunnell et al., 1983, 1985; Torsvall et al., 1984). Clearly, the increases in HR are partly due to increased physiological demands during exercise. However, during sleep, while the body is basically stationary, changes in HR would be attributable to alterations in autonomic activity. Thus, measures of HR are extremely useful tools for investigating autonomic changes during sleep. 
As a continuously varying measure, $\mathrm{HR}$ can be assessed over a wide range of time frames. Changes in HR variability (HRV) itself have also been studied (Ako et al., 2003), and have only recently become the focused measures of interest.

It has been shown that chronic exercise affects HR and HRV (Dixon et al., 1992; Furlan et al., 1993; Routledge et al., 2010). Recent meta-analysis of the effect of exercise therapy on HRV (Sandercock et al., 2005) indicates that habitual exercise enhances vagal modulation, resulting in bradycardia. Such vagal modulation could reflect enhancement of parasympathetic control, which could, thus improve both sleep and mood.

Only one extensive study by Catai et al. (2002) has examined exercise and HRV during sleep. There, 10 young and 7 middle-aged sedentary subjects were recruited to a 3-month aerobic training program, during which HRV was examined during sleep. Unfortunately, sleep EEG was not recorded. No changes in HRV were found after 3-months of the aerobic training program. Recently, Myllymäki et al. $(2011,2012)$ published two papers focusing exclusively on cardiac autonomic function during sleep. Their studies were designed to observe the acute effects of single bouts of exercise on later sleep. As expected, they confirmed HR elevations, but could not find significant HRV changes even during sleep that occurred shortly after vigorous exercise. Since there were no significant elevations of either sympathetic or parasympathetic nervous system activities, the authors concluded that vigorous late-night exercise does not disturb sleep quality.

Recently we studied an effect of vigorous late-night exercise on night sleep. We analyzed HRV in NREM and REM sleep separately for each sleep cycle, and found that the sympathetic nervous system activity parameter HF/LF was significantly elevated during the second NREM cycle (Shioda et al., 2012).

Thus, it is probably premature to make firm conclusions based on the current lack of consistent HRV responses during sleep after exercise. Although the studies to date were specifically aimed at examining the exercise effects on the cardiac system, they still reflect the early efforts in this field of study. As in the instance of the early studies of exercise effects on sleep EEG, examinations of sleep-specific exercise effects on the cardiac system may also require careful controls for relevant covariates such as the aerobic/anaerobic qualities, the intensity and timing of exercise, and the subjects' fitness status.

\section{EXERCISE EFFECTS ON ENDOCRINE FUNCTION DURING SLEEP}

In early studies, two research groups examined exercise effects on endocrinological changes during sleep. Their interests were mainly driven by changes in growth hormone $(\mathrm{GH})$ secretion, which was already known to be closely related to sleep (Takahashi et al., 1968). Once again, as for other sleep related physiological studies, early findings were contradictory. Adamson et al. (1974) found that GH increased during sleep after exercise, while Zir et al. (1971) had not. However, Adamson et al. also found decreased cortisol secretion after exercise, while later studies found increases in cortisol (Torsvall et al., 1984) and urinary norepinephrine excretion (Bunnell et al., 1985).

In a relatively new and extensive study, Kanaley et al. (1997) examined GH secretion patterns with repeated bouts of aerobic exercise, and then the amount of GH release during the following night's sleep. Compared to its release after a single bout of exercise, GH release was increased by repeated bouts of aerobic exercise, but there were no changes in the pattern of GH release during sleep.

More research needs to be done on the effects of exercise on hormonal levels during sleep. As with the effects of exercise on sleep EEG, sleep-specific endocrine responses to exercise have to be studied intensely, taking into account various factors such as exercise type, intensity, timing, subjects, and differences between acute and chronic exercise.

\section{EXERCISE EFFECTS ON METABOLIC FUNCTIONS DURING SLEEP}

There has been increasing interest in the relationships between sleep and metabolic functioning (Morselli et al., 2012), following epidemiological investigations detailing the relationships between sleep loss, obesity, and diabetes (e.g., Knutson and Van Cauter, 2008). An extensive experimental study (Van Cauter et al., 1991) indicated that blood glucose level is high during sleep, mainly because of the less active state of the whole brain (although some sleep-specific functions can drive local brain areas to high levels of activity), which is the largest glucose-consuming organ.

However, almost no data has been obtained to examine exercise effects on subsequent metabolic functions during sleep. As described above, since there have been reports that vigorous latenight exercise increases HR during sleep; it is reasonable to expect that there should also be some changes in metabolic rate during sleep. Such studies are not only important with regard to emerging global health problems, but they would be of special interest for the therapy and prevention of Type II diabetes.

\section{EXERCISE EFFECTS ON MOOD}

It has long been known (Cureton, 1963; Folkins, 1976) that exercise has beneficial effects on mood. Recent extensive studies on the therapeutic effects of exercise for depressive symptoms indicate that exercise could become an adjunctive, or possibly alternative therapy for depression (Blumenthal et al., 1999; Dunn et al., 2005). Since sleep disturbance is one of the core symptoms in depression, and because improved depressive symptoms are also associated with improved sleep quality, it is plausible that the effects of chronic exercise in improving sleep quality could be a relevant mechanism.

Models for a biological basis for such a therapeutic mechanism have been studied. Several hypothetical mechanisms have been suggested, including monoamine activation, hypothalamuspituitary-adrenal activation, or brain derived neurotrophic factor (BDNF) mediated changes (Brosse et al., 2002). Among these, a BDNF hypothesis has recently drawn particular research attention. It has been well established that exercise increases BDNF concentration in the brain (Vaynman and Gomez-Pinilla, 2005). There have also been reports of a therapeutic effect of BDNF for depressive symptoms (Vaynman and Gomez-Pinilla, 2005; Zheng et al., 2006; Sarris et al., 2008; Heyman et al., 2011; Toups et al., 2011). Recently, serum BDNF in depressive patients was also found to normalize after exercise (Laske et al., 2010). 


\section{EXERCISE EFFECTS ON SLEEP: FUTURE DIRECTIONS}

Reviewing the past studies of exercise effects on sleep suggests several pathways through which exercise could produce effects on sleep quality (Figure 2). Those pathways could be classified into three categories based on acute effects, acute, but with subsequent chronic effects, and effects arising after patterns of chronic exercise.

Conceivable acute effects would include CNS fatigue, BT elevation, or $\mathrm{HR} / \mathrm{HRV}$ changes. Whether fatiguing physical exercise causes a directly analogous CNS fatigue is an ongoing question. Even so the effect should not be significant, given the inconsistent results obtained when the effects of one bout of acute exercise on SWS are examined. The hypothesis that BT elevation itself enhances SWS is theoretically interesting, but should be studied in more detail, particularly in humans, especially to compare the effects of passive body heating with those after late-night exercise. Most studies have confirmed HR elevation during subsequent sleep, when HR is elevated during exercise. In addition to HR elevations, sympathetic enhancement was also reported. However, whether these changes have disturbing effects on sleep also needs further study.

Some acute endocrine and metabolic effects of exercise may produce chronic effects when exercise becomes habitual. Habitual repetitions of acute elevations in glucose metabolism, GH, and BDNF releases are conceivable mechanisms that could lead to alterations in sleep. Late-night exercise could lower the glucose level during sleep, although we still do not know its effects on sleep. Even without long-term adaptation, exercise related glucose reductions could have a favorable effect for Type II diabetes, as glucose levels are typically relatively high during sleep (Van Cauter et al., 1991). Similarly, exercise increases daytime GH release, which might directly affect sleep quality. However, since GH secretion is closely related to SWS (Takahashi et al., 1968), the long-term effect of repeatedly increased GH releases over $24 \mathrm{~h}$ could alter sleep quality.

It is also known that exercise acutely enhances BDNF production. It has not yet been established whether BDNF directly alters sleep quality or not. However, the anti-depressant effects of exercise have recently been well studied, and improved mood

\section{REFERENCES}

Adamson, L., Hunter, W. M., Ogunremi, O. O., Oswald, I., and PercyRobb, I. W. (1974). Growth hormone increase during sleep after daytime exercise. J. Endocrinol. 62, 473-478.

Ako, M., Kawara, T., Uchida, S., Miyazaki, S., Nishihara, K., Mukai, J., Hirao, K., Ako, J., and Okubo, Y. (2003). Correlation between electroencephalography and heart rate variability during sleep. Psychiatry Clin. Neurosci. 57, 59-65.

Aserinsky, E., and Kleitman, N. (1953). Regularly occurring periods of eye motility, and concomitant phenomena, during sleep. Science 118, 273-274.

Baekeland, F., and Lasky, R. (1966). Exercise and sleep patterns in college athletes. Percept. Mot. Skills 23, 1203-1207.

Blumenthal, J. A., Babyak, M. A., Moore, K. A., Craighead, W. E., Herman, S., Khatri, P., Waugh, R., Napolitano, M. A., Forman, L. M., Appelbaum, M., Doraiswamy, P. M., and Krishnan, K. R. (1999). Effects of exercise training on older patients with major depression. Arch. Intern. Med. 159, 2349-2356.

Brosse, A. L., Sheets, E. S., Lett, H. S., and Blumenthal, J. A. (2002). Exercise and the treatment of clinical depression in adults: recent findings and future directions. Sports Med. 32, 741-760.

Bunnell, D. E., Bevier, W. C., and Horvath, S. M. (1983). Nocturnal sleep, cardiovascular function, and adrenal activity

attributable to a BDNF elevation attributable to exercise could indirectly improve sleep quality. Such pathway relationships between acute and chronic effects of exercise and alterations in sleep should be examined in the future studies.

One certain beneficial effect of habitual exercise is improved health. Besides, some psychological benefits (which should not be ignored) as well as their interactions with acute and chronic physiological alterations over time, could themselves improve sleep. The addition of non-invasive long-term physiological measurements that do not disturb sleep, such as measures of sympathetic predominance measured by HRV, can reveal such sleep promoting alterations. Studies have shown that exercise definitely changes body composition (Garrow and Summerbell, 1995) and cardiovascular fitness levels (Warburton et al., 2006). Meta-analyses (Figure 1) indicate that chronic exercise yields more stable and persistent improvements in sleep. These benefits likely reflect the summed and interacting benefits of numerous physiological alterations by chronic exercise, which directly and indirectly affect sleep quality (Figure 2).

The lifelong, apparently passive, automatic homeostatic cycling between wakefulness and sleep is influenced by several factors including physical activity, which also involves the prior activity in a large network of neurons of the CNS. These activities influence the global tendency of the CNS for sleep-wake oscillation (Kumar, 2010). Exercise could be a robust stimulus for the auto-regulatory global phenomenon which affects the entirety of the sleeper's physiological mechanisms. Appropriate amounts of exercise could alter those mechanisms in a preferable direction, toward restoration of the levels humans originally needed and adapted to for living, offsetting some important health consequences attributable to conveniences in modern society.

\section{ACKNOWLEDGMENTS}

This work was supported in part by Global COE Program (Sport Sciences for the Promotion of Active Life) MEXT Japan, and KIBANKEISEI, MEXT Japan. The authors thank Dr. Thomas Maloney for kind and detailed editing of the manuscript.

following maximum-capacity exercise. Electroencephalogr. Clin. Neurophysiol. 56, 186-189.

Bunnell, D. E., Bevier, W. C., and Horvath, S. M. (1985). Effects of exhaustive submaximal exercise on cardiovascular function during sleep. $J$. Appl. Physiol. 58, 1909-1913.

Catai, A. M., Chacon-Mikahil, M. P. T., Martinelli, F. S., Forti, V. A. M., Silva, E., Golfetti, R., Martins, L. E. B., Szrajer, J. S., Wanderley, J. S., LimaFilho, E. C., Milan, L. A., MarinNeto, J. A., Maciel, B. C., and GalloJunior, L. (2002). Effects of aerobic exercise training on heart rate variability during wakefulness and sleep and cardiorespiratory responses of young and middle-aged healthy men. Braz. J. Med. Biol. Res. 35, 741-752.
Cureton, T. K. (1963). Improvement of psychological states by means of exercise-fitness programs. J. Assoc. Phys. Ment. Rehabil. 17, 14-17.

Davis, H., Davis, P. H., Loomis, A. L., Harvey, E. N., and Hobart, G. (1937). Changes in human brain potentials during the onset of sleep. Science 86, 448-450.

Dixon, E. M., Kamath, M. V., and McCartney, N., and Fallen, E. L. (1992). Neural regulation of heart rate variability in endurance athlete and sedentary controls. Cardiovasc. Res. 26, 713-719.

Dunn, A. L., Trivedi, M. H., Kampert, J. B., Clark, C. G., and Chambliss, H. O. (2005). Exercise treatment for depression: efficacy and dose response. Am. J. Prev. Med. 28, $1-8$. 
Feinberg, I., March, J. D., Fein, G., Floyd, T. C., Walker, J. M., and Price, L. (1978). Period and amplitude analysis of $0.5-3 \mathrm{c} / \mathrm{sec}$ activity in NREM sleep of young adults. Electroencephalogr. Clin. Neurophysiol. $44,202-213$.

Flausino, N. H., Da Silva Prado, J. M., de Queiroz, S. S., Tufik, S., and De Mello, M. T. (2012). Physical exercise performed before bedtime improves the sleep pattern of healthy young good sleepers. Psychophysiology 49, 186-192.

Folkins, C. H. (1976). Effects of physical training on mood. J. Clin. Psychol. $32,385-388$.

Furlan, R., Piazza, S., Dell'Orto, S., Gentile, E., Cerutti, S., Pagani, M., and Malliani, A. (1993). Early and late effects of exercise and athletic training on neural mechanisms controlling heart rate. Cardiovasc. Res. 27, 482-488.

Garrow, J. S., and Summerbell, C. D. (1995). Meta-analysis: effect of exercise, with or without dieting, on the body composition of overweight subjects. Eur. J. Clin. Nutr. 40, 1-10.

Heyman, E., Gamelin, F.-X., Goekint, M., Piscitelli, F., Roelands, B., Leclair, E., Di Marzo, V., and Meeusen, R. (2011). Intense exercise increases circulating endocannabinoid and BDNF levels in humanspossible implications for reward and depression. Psychoneuroendocrinology. Available at: http://dx.doi.org/ 10.1016/j.psyneuen.2011.09.017

Horne, J. A., and Staff, L. H. E. (1983). Exercise and sleep: body-heating effects. Sleep 6, 36-46.

Kajimura, N., Uchiyama, M., Takayama, Y., Uchida, S., Uema, T., Kato, M., Sekimoto, M., Watanabe, T., Nakajima, T., Horikoshi, S., Ogawa, K., Nishikawa, M., Hiroki, M., Kudo, Y., Matsuda, H., Okawa, M., and Takahashi, K. (1999). Activity of midbrain reticular formation and neocortex during the progression of human non-rapid eye movement sleep. J. Neurosci. 19, 10065-10073.

Kanaley, J. A., Weltman, J. Y., Veldhuis, J. D., Rogol, A. D., Hartman, M. L., and Weltman, A. (1997). Human growth hormone response to repeated bouts of aerobic exercise. Electroencephalogr. Clin. Neurophysiol. 83, 1756-1761.
Knutson, K. L., and Van Cauter, E. (2008). Associations between sleep loss and increased risk of obesity and diabetes. Ann. N. Y. Acad. Sci. 1129, 287-304.

Kubitz, K. A., Landers, D. M., Petruzzello, S. J., and Han, M. (1996). The effects of acute and chronic exercise on sleep a metaanalytic review. Sports Med. 21, 277-291.

Kumar, V. M. (2004). Body temperature and sleep: are they controlled by the same mechanism? Sleep Biol. Rhythms 2, 103-124.

Kumar, V. M. (2010). Sleep is neither a passive nor an active phenomenon. Sleep Biol. Rhythms 8, 163-139.

Laske, C., Banschbach, S., Stransky, E., Bosch, S., Straten, G., MacHann, J., Fritsche, A., Hipp, A., Niess, A., and Eschweiler, G. W. (2010). Exercise-induced normalization of decreased BDNF serum concentration in elderly women with remitted major depression. Int. J. Neuropsychopharmacol. 13, 595-602.

McGinty, D., and Szymusiak, R. (1990). Keeping cool: a hypothesis about the mechanisms and functions of slow-wave sleep. Trends Neurosci. 13, 480-487.

Morselli, L. L., Guyon, A., and Spiegel, K. (2012). Sleep and metabolic function. Pflügers Arch. Eur. J. Physiol. 463, 139-160.

Myllymäki, T., Kyröläinen, H., Savolainen, K., Hokka, L., Jakonen, R., Juuti, T., Martinmäki, K., Kaartinen, J., Kinnunen, M.-L., and Rusko, H. (2011). Effects of vigorous late-night exercise on sleep quality and cardiac autonomic activity. $J$. Sleep Res. 20, 146-153.

Myllymäki, T., Rusko, H., Syväoja, H., Juuti, T., Kinnunen, M. L., and Kyröläinen, H. (2012). Effects of exercise intensity and duration on nocturnal heart rate variability and sleep quality. Eur. J. Appl. Physiol. 112, 801-809.

Pedersen, B. K., and Saltin, B. (2006). Evidence for prescribing exercise as therapy in chronic disease. Scand. J. Med. Sci. Sports 16(Suppl. 1), 3-63.

Rechtschaffen, A., and Kales, A. (eds). (1968). A Manual of Standardized Terminology, Techniques and Scoring System of Sleep Stages in Human
Subjects. Los Angeles: Brain Information Service/Brain Research Institute, University of California.

Roberts, C. K., and Barnard, R. J. (2005). Effects of exercise and diet on chronic disease. J. Appl. Physiol. 98, 3-30.

Roussel, B., and Buguet, A. (1982). Changes in human heart rate during sleep following daily physical exercise. Eur. J. Appl. Physiol. Occup. Physiol. 49, 409-416.

Routledge, F. S., Campbell, T. S. McFetridge-Durdle, J. A., and Bacon, S. L. (2010). Improvements in heart rate variability with exercise therapy. Can. J. Cardiol. 26, 303-312.

Sandercock, G. R. H., Bromley, P. D., and Brodie, D. A. (2005). Effects of exercise on heart rate variability: inferences from meta-analysis. Med. Sci. Sports Exerc. 37, 433-439.

Sarris, J., Kavanagh, D. J., and Newton, R. (2008). Depression and exercise. J. Complement. Med. 7, 48-53.

Shioda, K., Goto, K., and Uchida, S. (2012). The effect of acute highintensity exercise on following night sleep. J. Japanese Soc. Clin. Sports Med. (in print).

Takahashi, Y., Kipnis, D. M., and Daughaday, W. H. (1968). Growth hormone secretion during sleep. J. Clin. Invest. 47, 2079-2090

Torsvall, L., Akerstedt, T., and Lindbeck, G. (1984). Effects on sleep stages and EEG power density of different degrees of exercise in fit subjects. Electroencephalogr. Clin. Neurophysiol. 57, 347-353.

Toups, M. S. P., Greer, T. L., Kurian, B. T., Grannemann, B. D., Carmody, T. J., Huebinger, R., Rethorst, C., and Trivedi, M. H. (2011). Effects of serum brain derived neurotrophic factor on exercise augmentation treatment of depression. J. Psychiatr. Res. 45, 1301-1306.

Uchida, S., Feinberg, I., March, J. D. Atsumi, Y., and Maloney, T. (1999). A comparison of period amplitude analysis and FFT power spectral analysis of all-night human sleep EEG. Physiol. Behav. 67, 121-131.

Van Cauter, E., Blackman, J. D., Roland, D., Spire, J.-P., Refetoff, S., and Polonsky, K. S. (1991). Modulation of glucose regulation and insulin secretion by circadian rhythmicity and sleep. J. Clin. Invest. 88, 934-942.
Vaynman, S., and Gomez-Pinilla, F. (2005). License to run: exercise impacts functional plasticity in the intact and injured central nervous system by using neurotrophins. Neurorehabil. Neural. Repair 19, 283-295.

Walker, J. M., Floyd, T. C., Fein, G., Cavness, C., Lualhati, R., and Feinberg, I. (1978). Effects of exercise on sleep. J. Appl. Physiol. 44, 945-951.

Warburton, D. E. R., Nicol, C. W., and Bredin, S. S. D. (2006). Health benefits of physical activity: the evidence. Can. Med. Assoc. J. 174 801-809.

Youngstedt, S. D., O'Connor, P. J., and Dishman, R. K. (1997). The effects of acute exercise on sleep: a quantitative synthesis. Sleep 20, 203-214.

Zheng, H., Liu, Y., Li, W., Yang, B., Chen, D., Wang, X., Jiang, Z., Wang, H., Wang, Z., Cornelisson, G., and Halberg, F. (2006). Beneficial effects of exercise and its molecular mechanisms on depression in rats. Behav. Brain Res. 168, 47-55.

Zir, L. M., Smith, R. A., and Parker, D. C. (1971). Human growth hormone release in sleep: effect of daytime exercise. J. Clin. Endocrinol. Metab. 32, 662-665.

Conflict of Interest Statement: The authors declare that the research was conducted in the absence of any commercial or financial relationships that could be construed as a potential conflict of interest.

Received: 28 February 2012; accepted: 14 March 2012; published online: 02 April 2012.

Citation: Uchida S, Shioda K, Morita $Y$, Kubota C, Ganeko $M$ and Takeda $N$ (2012) Exercise effects on sleep physiology. Front. Neur. 3:48. doi: 10.3389/fneur.2012.00048

This article was submitted to Frontiers in Sleep and Chronobiology, a specialty of Frontiers in Neurology.

Copyright (c) 2012 Uchida, Shioda, Morita, Kubota, Ganeko and Takeda. This is an open-access article distributed under the terms of the Creative Commons Attribution Non Commercial License, which permits non-commercial use, distribution, and reproduction in other forums, provided the original authors and source are credited. 